\title{
Masculinidades en el rap. Por una construcción del rap chileno actual
}

\author{
Germán Alcalde \\ Pontificia Universidad Católica de Chile \\ galcalde2@uc.cl
}

Hace algún tiempo Snoop Dog, famoso rapero estadounidense, dijo que su compatriota Frank Ocean no era rapero sino cantante debido a que era homosexual, argumentando que el rap era necesariamente masculino (Radio Bío-bío). En Chile, La Celda de Bob, blog periodístico relacionado con el mundo del hip-hop, recogió la historia de Moises (La celda de Bob, "SIGMUND KROYS"), una persona que en su juventud perteneció a esta cultura urbana pero que luego se desligó de la misma, en parte por sentirse excluido por su homosexualidad. Estas historias hacen necesaria una pregunta ¿Qué relación hay entre la cultura hip-hop, incluyendo al rap, y la masculinidad? Este breve trabajo intentará dar una posible respuesta en el contexto del rap santiaguino de la novísima escuela -la generación que está en la cumbre hoy- con el fin de conocer cómo una de las sub-culturas juveniles urbanas más influyentes en nuestros tiempos se posiciona frente a este tema para construir su identidad.

Para este trabajo se tomará como base la novísima escuela debido a que, al ser heredera de las otras escuelas más antiguas y, en cierta medida, del rap estadounidense, el rap chileno de la novísima escuela es, a pesar de sus particularidades, el perfecto ejemplo del rap en general, por lo que los análisis y conclusiones de este trabajo pueden ser fácilmente tomadas como base para ampliar la investigación a otras escuelas de rap en otros países u otros tiempos. Para el desarrollo de la investigación revisaré, a modo general, el contexto y las prácticas del hip-hop nacional, con el fin de entender la forma en que este se construye, para luego analizar distintas canciones buscando entender, a través de la voz de los propios raperos, cómo se construyen las masculinidades dentro del hip-hop.

Las preguntas necesarias que nacen a partir de lo anterior tienen que ver con las razones y las consecuencias de las masculinidades dentro de la identidad del hip-hop; hay que preguntarse el por qué son importantes para la construcción del movimiento y que función cumplen -si es que cumplen alguna- para lo que tenemos que comprender cómo se articulan. Además, es necesario preguntarse por las consecuencias que traen las masculinidades en el rap, con reflexiones relacionadas con el machismo y la homofobia que puede existir en el hip-hop, o con la forma en la que las raperas femeninas se instauran dentro de este esquema. 


\section{¿Qué es el rap?}

\section{Contexto de creación y expansión}

Para adentrarse en el mundo del hip-hop lo primero que hay que hacer es conocer el contexto ${ }^{1}$ en el cual este se creó, ya que "saber de hip hop, de sus orígenes y su historia es muy importante para la mayoría de los hiphoperos" (Codocedo 127), lo que se traduce de forma evidente en las propias poéticas de los artistas, pues estos se ven reflejados en sus antecesores. ${ }^{2}$ El hip-hop es una cultura urbana creada "a comienzos de la década del 70 en los barrios pobres de Nueva York" (Asfura 1011) en un contexto de "ilegalidad, falta de empleo, un creciente mercado de drogas, abandono municipal y la floración de cientos de 'pandillas juveniles' que solucionaban sus problemas o ajustes de cuentas a través de la violencia" (Poch Plá 74).

Dentro de esta cultura urbana, que es "una especie de recipiente cultural en que entran y salen diversas expresiones artísticas, sociales y valóricas” (Poch Plá 103), se encuentran los llamados "cuatro elementos" o "ramas": manifestaciones artísticas relacionadas con la cultura. ${ }^{3}$ Estas son: el break dance-baile callejero-, el graffiti -arte pictórico en las paredes de las ciudades-, el disk jockey o Dj-composición musical del rap- y el Mcing -líricas del rap. Es por lo anterior que, cuando hable de hip-hop será en referencia a toda la cultura urbana mencionada, mientras que, cuando hable de rap, me centraré únicamente a la música escrita por un $M c$ o rapero -persona que practica el Mcing - y compuesta por un Dj o Disk jokey, tomándola como parte del hip-hop.

Siguiendo con la creación del hip-hop; en este contexto de desigualdad socio-económica-racial se creó "la necesidad de buscar nuevos espacios de socialización” (Espita 21) lo que dio paso a las llamadas block parties; "eventos constituidos no solo como espacios de dispersión, sino como procesos de relación entre iguales" (Espita 21). En estos eventos se buscó un "espíritu transformador que [...] llevaría a organizarse para cambiar este entorno viciado” (Poch Plá 327), lo que sentó las bases para la conformación de "una comunidad propia, basada en un nuevo tipo de sentimiento y energía” (Quitzow). Así los primeros hiphoperos "comenzaron a buscar soluciones a sus problemas en un lugar que no fueran las políticas de gobierno ni los programas asistenciales de las fundaciones de beneficencia” (Poch Plá

1 Hablo de contexto y no de historia ya que este trabajo busca centrarse en un análisis textual del rap y no en una revisión historiográfica del hip-hop. Para una revisión más completa revisar Poch Plá, Codocedo, Asfura y Quitzow.

2 Esto se da porque, a pesar de las diferencias culturales, los estigmas vividos por los primeros raperos en los años 70 se homologan con las realidades de los raperos chilenos actuales; como menciona Codocedo, para ser rapero hay que "saber que los primeros hiphoperos fueron negros oprimidos, [y hay que tener] una cierta identificación con esta condición” (129).

3 Para más información revisar Poch Plá o Codocedo. 


\section{Adiós a las armas}

Despatriarcar América desde la cultura

74), lo que transformó al hip-hop en "una alternativa al círculo de la violencia, y en una expresión cultural que se comenzó a reproducir socialmente” (Poch Plá 75).

Durante las siguientes décadas el hip-hop se expandió fuera de Estados Unidos, hasta que, a finales de los 80 y principios de los 90, llegó a Chile, siendo las primeras canciones de rap grabadas profesionalmente producidas hacia finales de la dictadura. ${ }^{4}$ Fue en este contexto "con la sociedad alejándose de las ideologías del pasado, y con un Santiago progresivamente marcado por la segregación económica y una mentalidad individualista y consumista, [que] el hip hop representó una aproximación fresca y novedosa a las cambiantes realidades de Chile" (Quitzow). Es necesario recalcar que, en nuestro país, el hip-hop se arraigó "no debido a alguna poderosa estrategia de marketing, sino gracias a su atractivo para una juventud deprivada en las poblaciones de Santiago" (Quitzow) gracias a la identificación que sentían los jóvenes urbanos populares con este movimiento y sus cultores originales.

\section{Contexto actual}

Ya han pasado dos décadas y tres generaciones -conocidas como la Vieja Escuela (con grupos como De Kiruza y los Panteras Negras), la Nueva Escuela (liderada por Tiro de gracias y Makiza) y la Novísima, que considera "producciones y registros desde el año 2000” (Asfura 19)- desde la llegada del hip-hop a Chile. Actualmente el hip-hop "es un fenómeno ampliamente extendido por muchas poblaciones y comunas de Santiago" (Codocedo 4) e incluso en otras ciudades de nuestro país. Es gracias a lo anterior que han variado los estilos y que, hoy en día, existan tantas formas de hacer rap como raperos en la calle. Sin embargo, a pesar de la variedad de propuestas, todos los raperos y hiphoperos parecen mantener cierto sistema valórico, estudiado por la antropóloga Paula Codocedo, el cual se analizará a continuación.

Codocedo, a partir de entrevistas realizadas a jóvenes hiphoperos de Santiago, afirma que existe un sistema valórico hip-hop y divide los valores en dos grupos; los compartidos por todos y los no compartidos por todos. Según los resultados se encuentran entre los valores compartidos el carácter lúdico del hip-hop (es para pasarlo bien), la competencia - con uno mismo y los demás- como una forma de superarse, la originalidad, la práctica, cierto tipo de mesura (ser "piola" no "alumbrado”), la valoración de la historia del hip-hop, y el rechazo a la visión del hip-hop como moda. Por su parte, entre los valores no compartidos por todos se encuen-

4 "Algo está pasando" del grupo De Kiruza, considerada la primera canción de rap grabada profesionalmente, fue lanzada en 1988, poco antes del final de la dictadura militar. 
tran ser anti-sistémico, anti-comercial, contestatario, la idea de ver al hip-hop como un estilo de vida y la necesidad de ser artista profesional.

A partir de estos resultados, que intentan ser un fiel reflejo de la imagen propia que tienen los hiphoperos santiaguinos, se pueden sacar algunas conclusiones: primero, es notable que no esté entre los valores la necesidad de pertenecer a cierto estrato socio-económico, como podría pensarse, sino que parece ser más importante respetar su historia, desde donde se desprende el respeto a los sectores que viven una situación similar a la de los primero hiphoperos. Segundo, destaca que los valores compartidos por todos se enfoquen en la propia imagen y en la relación con los demás hiphoperos, mientras que los compartidos por algunos sean valores relacionados con la sociedad, dando la idea de que hay más de un hip-hop y, por lo tanto, más de un rap, como se verá en el siguiente apartado. Por último, me gustaría recalcar la idea de la competencia como forma de superación, punto que será esencial para comprender cierto tipo de rap.

\section{Comunidad y competencia, hacia una posible clasificación del rap}

Ya entendiendo un poco desde donde se posiciona el hip-hop nacional quisiera detenerme en el rap y sus distintas formas. Como mencioné existen tantas maneras de hacer rap como raperos, pero se pueden englobar todos estos "estilos" en dos grandes secciones: el rap hardcore y el rap consciente. El rap hardcore se entiende como "lo que representa el vacile, la calle, la competi[ción] [mientras que al rap consciente] se le ha dado un contexto frontalmente político más que de interés social global" (La celda de Bob, "Mamá, Papá, soy rapero”). Las definiciones anteriores parecen no convencer al entrevistador debido a que estas se usan de formas "radicales, determinantes, y a la vez tan alejadas de la realidad" (La celda de Bob, "Mamá, Papá, soy rapero"), haciendo que los cultores tomen partido por una de ellas y renieguen de la otra. En este sentido es que haré algunas consideraciones para dar cuenta de estos conceptos sin, como dice Güissario Patiño, alejarme de lo que realmente sucede en el rap chileno.

Como ya se ha mencionado, el hip-hop nació en un contexto de búsqueda de comunidad entre la violencia; una violencia que venía tanto desde afuera de la comunidad, por parte de los sectores hegemónicos, como desde adentro, de la mano de las llamadas "pandillas juveniles". Es por lo anterior que, entre las propuestas dadas en el hip-hop original, los primeros Dj's "comenzaron a plantear que los problemas de apropiación territorial y de índole personal debían resolverse mediante batallas de carácter artístico y no en enfrentamientos con armas o golpes que pudieran causar daño" (Poch Plá 75). Esto creó un doble espíritu que se manifiesta en estos dos estilos; por un lado, el rap consciente se creó debido a que se concibió al conocimiento (político, social, moral) como la base necesaria para la 


\section{Adiós a las armas}

Despatriarcar América desde la cultura

creación de una comunidad y, por el otro, el rap hardcore nació de la sublimación de la violencia, ejercida por los mismos hiphoperos, que se vivía en el contexto ya explicado.

Estos dos estilos, que parecen ser opuestos, no lo son completamente (de ahí la crítica de Patiño a que no representa la realidad). Lo anterior porque: en primer lugar, la mayoría de los raperos no son ni hardcore ni conscientes, sino que mezclan elementos de ambos espíritus en sus poéticas, incluso complementándolos en ciertas ocasiones. ${ }^{5}$ En segundo lugar, hay elementos hardcore que nacen de la necesidad de comunidad, como lo es el "vacile" (fiesta) y elementos del rap consciente que nacen de la competencia. Por último, existen características de los raperos que son imposibles de encasillar en solo un estilo, como lo es la superación, mencionada anteriormente como valor, que puede verse como hardcore al haber otro al cual superar o como consciente al ser auto superación. Es por las razones anteriores que, en lugar de subgéneros, me refiero a ellos como estilos o espíritus, haciendo referencia a que, a pesar de su posible contradicción, pueden coexistir e incluso complementarse dentro de las poéticas de los artistas.

\section{Masculinidades en el rap}

\section{Rap masculino}

¿Cómo entra entonces la masculinidad en el hip-hop y su historia? debido a su pasado y a su identidad es necesario entender que no solo el género está en juego cuando se habla de la masculinidad rapera, sino que también se deben tomar en cuenta otros ejes de la identidad como son la raza y la clase social. Con base a lo anterior se puede posicionar la masculinidad rapera dentro de la masculinidad marginal descrita por Connell, ya que, en la configuración del rap la posición de marginados juega un rol fundamental.

Ahora, cabe preguntarse ¿por qué debe ser masculino el rap? Tentativamente se puede pensar que esto es para equilibrar su carencia de poder socio-económico aprovechándose del dividendo patriarcal que rige nuestra cultura, es decir, al estar en desventaja por su posición marginal, el rapero suple esa falta con un exceso de masculinidad. Lo anterior, sin dejar de ser cierto, no alcanza a vislumbrar la profundidad del fenómeno, por lo que es necesario ahondar en la forma en la que los raperos buscan manifestar sus masculinidades, tanto en el rap consciente como en el rap hardcore.

5 Destaca la frase de Gran Rah "¿Querí rap hardcore? ¿Querían consciencia social? La vida es hardcore hermano" (Gran Rah, "Vida Hardcore"). 
Para comprender la identidad rapera, y, por lo tanto, su masculinidad, se debe conocer su posición dentro de la cultura, ya no histórica, sino que espacial y metafóricamente. Los propios "hiphoperos se consideran a sí mismos como artistas populares, artistas que desarrollan su arte en las calles de las ciudades, en las poblaciones" (Codocedo 108), por lo que la construcción del sujeto rapero debe verse en esta doble vertiente de marginalidad y urbanidad como una sola característica, pues la marginalidad ejercida en los sujetos es la propia de la ciudad y, a la vez, la urbanidad y sus representaciones llevan consigo la concepción de una sector marginado dentro de la ciudad más que una simple bohemia.

Como señalé anteriormente, el rap nació en una cuna de marginalidad y exclusión, lo que ha marcado profundamente a sus sujetos. Teniendo en cuenta que para ser rapero hay que "saber que los primeros hiphoperos fueron negros oprimidos, [y hay que ligarse] a una cierta identificación con esta condición” (Codocedo 129), se puede entender que hoy el rap pertenezca principalmente a los marginados, que son precisamente los mencionados por Connell cuando da cuenta de las masculinidades marginales en Estados Unidos, siendo entonces sus mayores ejemplos los negros y los pobres. Es necesario recalcar que dentro de esta definición se excluye a otros tipos de marginación como los homosexuales que pasan a ser parte de los subordinados, ya que están ligados con el imaginario femenino (Connell). En Chile esta identificación con el sujeto marginal estadounidense se transforma para dar cuenta de nuestra propia realidad; "lo negro en EEUU se asimila a lo indígena, a la pobreza y a la juventud en Chile y en las poblaciones" (Codocedo 131).

Pero la marginalidad no es la única característica que marcó los inicios y el desarrollo del hip-hop; los primeros raperos fueron sujetos altamente urbanos sumergidos en la modernidad capitalista presente en Estados Unidos. Lo anterior lleva a que la identificación con los primeros raperos mencionada anteriormente se vincule "con la identificación con el territorio: el barrio, la población" (Codocedo 129), es decir, los espacios del hip-hop son los lugares de la ciudad que se encuentran marginados; no es una urbe prometedora llena de luces, sino el espacio de la urbe en donde los marginados viven excluidos del resto de la población urbana.

En este contexto podemos entender uno de los lugares más simbólicos del rap; la calle, que es la metáfora de la dureza y frialdad de la urbanidad-marginal, que, sin embargo, se llena de luz pues es el lugar de los raperos - dice Hordatoj "vi raperos en la calle, en el cielo las estrellas" (Hordatoj, "Un segundo"). Esta identificación también inunda otros lugares urbanos como el barrio y la comuna, los cuales están a la vez dotados de una relación personal con el rapero pues es el lugar "que me vio crecer" (Chyste Mc en Da Killtros, "Uno punto nueve barrio”). Pero ¿qué significa ser parte de esta urbanidad-marginal? representa por una parte un conocimiento especial sobre la realidad -Jonas Sanche dice "la educación que tengo la dio la calle y mi abuela" (Jonas Sanche, "Estar asín") - y por otra, una fuerza singular que permite al sujeto rapero sobrevivir a las adversidades que trae la marginalidad, tanto 


\section{Adiós a las armas}

Despatriarcar América desde la cultura

visto en el riesgo que la calle representa -Gran rah dice de su barrio; "puente alto es la ciudad del homicidio" (Gran Rah, "Perro") - como en el dolor de ser marginado -Dous Mc dedica una canción sobre la exclusión al "que aguanta la presión/ el clásico prejuicio luego discriminación” (Dous Mc en Adickta Sinfonía, "Bicho raro").

Dentro de esta concepción de fuerza por pertenecer y ser sobreviviente de la calle encontramos la primera muestra de masculinidad; la marginalidad da fuerza al sujeto -“salir del barrio, me empuja a ser más fuerte” (Malcriaoh en Dania Neko, "Casquillos") - lo que lo transforma en un Soldado del ghetto como asegura Movimiento original, dando así al ser marginal una serie de connotaciones, que podrían asimilarse a lo militar, las cuales forman parte del imaginario masculino debido a su frialdad y su agresividad. Entonces, bajo esta concepción se llega a la idea de que mientras más marginal y resistente se sea, se es más masculino.

Siguiendo con lo anterior, el imaginario masculino frío y agresivo lleva ineludiblemente a la violencia, característica esencial del rap chileno y punto fundamental para entender las masculinidades raperas. La violencia en el rap se manifiesta principalmente mediante dos estrategias; el ataque hacia un tú y la propia proyección de una imagen violenta. Cuando se ataca a un tú, este se ve como un oyente imaginario que no se corresponde con el oyente ideal, sino que parece ser más el objeto lírico de las canciones -por ejemplo, al decir "no quiero saber más de ti/ aquí nadie es aprendiz de tus mentiras" (ARB en Adickta Sinfonía, "Vete ya") no se intenta atacar al que escucha la canción, sino que se le habla a un tú imaginario con dichas características. Este "tú" imaginario se construye como representante de ciertas personas (dentro del rap consciente podrían ser la clase política, los "cuicos" -personas de clase alta- o los "pacos" -carabineros-, mientras que en el rap hardcore se suele atacar a los raperos "falsos") contras las cuales se quiere ejercer la violencia.

Por su parte, cuando se busca resaltar la propia imagen violenta, esta se proyecta en algún rasgo que justifique la violencia - "como no hay respeto voy a darle por donde les duela" (Massibo en Bascur, "Resentidos sin gloria") - o que la demuestre dentro del rapero - "Voy a estrangular el track, con rimas sobre el clap [...] machucando al micro, escupiendo al anti-pop" (Jano A en Adickta Sinfonía, "Identidad propia”). También, al ser la calle símbolo de dureza y violencia, y al proyectarse dichas características en sus habitantes (los raperos), se potencia la imagen propia violenta, construyendo así la ultra-masculinidad característica de la masculinidad marginal presente en el rap santiaguino.

Una forma que se utiliza comúnmente para invalidar al otro y auto validarse, es decir, de atacar a un tú y de incrementar la propia imagen, es por medio del insulto homofóbico. Las masculinidades suelen construirse en un esquema diferencial con otras formas de construir el género, tomando distancia e incluso marginando a todo rasgo que se acerque a la femineidad, atacando entonces a la llamada mas- 
culinidad subordinada (Connell). Esto ocurre también en el rap chileno, pues los raperos suelen marcar su diferencia con estas otras masculinidades utilizando palabras como gay, maricón o poco hombre entre sus insultos - "no discrimino pero no soporto un gay en el equipo" (Aerstame, "Escupo ácido"), "el rec hip hop es mi pasión / la tuya sería enamorarme y casarte con un maricón” (Estrellas del porno, "Estilo sudaca represento"). Lo anterior tiene como consecuencia que el "tú" sea invalidado al estar excluido en el paradigma patriarcal y que el "yo" se vea validado pues, al insultar al otro por no ser lo suficientemente hombre, el rapero marca una diferencia que lo posiciona dentro de la masculinidad. Esto ha llevado a que a los homosexuales se les discrimine y exilie del hip-hop nacional, como se observa en el mencionado caso de Moisés relatado en el artículo "Mamá, Papá, soy rapero" de La celda de Bob.

Por otro lado, se puede notar que la violencia no solo se encuentra en el contenido del rap, sino que también en su forma; muchos raperos hardcore utilizan una voz rasposa y forzada (Como Omega el ctm o chr) la cual dota al rapero de una imagen monstruosa apoyando su agresividad. Esto también ocurre en las instrumentales, ya que muchas de estas son toscas y agresivas, con bajos y baterías golpeadas como principal componente (como se puede ver en las pistas de Adickta Sinfonía o De Killtros). Asimismo, el vocabulario utilizado en las canciones se funda en esta violencia siendo el garabato, muchas veces cómo insulto, parte fundamental del léxico del rap chileno.

Por último, me gustaría recalcar otra de las características clásicas de la masculinidad que está presente en el rap chileno, específicamente en la corriente hardcore. Dentro de las masculinidades podemos notar que "la bebida es un identificador de hombría" (Navarro) pues los hombres deben beber para demostrar su masculinidad frente a los amigos; el que no bebe no es hombre. Los raperos a partir de esto hacen constantes referencias a esta característica de la masculinidad por medio de la imagen del piante (vago, alcohólico, drogadicto, etc.), pues los propios raperos admiten y divulgan su consumo de alcohol y, dentro del mismo concepto, otras drogas como la mariguana, lo que lleva a muchos raperos a definirse como "chicha y drogo" (chr en Bascur, "El que la hace la paga") y a asegurar que no toman "alcohol pa' chiquillas" (Leviatán en Estrellas del porno, "Soy un borracho"). Lo anterior lleva a otra forma de construir la masculinidad, que es fácilmente homologable con el imaginario del hombre que llega tarde y ebrio a su casa después de ir de fiesta con los amigos, mientras la mujer se queda esperando sumisamente en la casa -dice chr "mi destreza es tremenda como la paciencia de mi vieja" (chr en Gran Rah, "Palabras mayores"). 


\section{Adiós a las armas}

Despatriarcar América desde la cultura

\section{Función de las masculinidades}

Como se vio, dentro del rap, la masculinidad ultra-agresiva y marginal se impone como un rasgo fundamental para la construcción de los sujetos, por lo que ahora toca preguntarse ¿qué sentido o utilidad tiene esto? Como dije anteriormente, se podría pensar que esto es para aprovecharse del dividendo patriarcal que la cultura ofrece para equilibrar la falta de otro motor de poder; el socio-económico-racial, pero esta respuesta peca de no estar completa, pues no responde el para qué sirve dicho poder ganado. Generalmente las masculinidades utilizan este poder para perpetuar su poder en la llamada lucha de géneros, pero en el rap sucede algo diferente; la masculinidad se utiliza como un instrumento que da poder para la lucha contra la discriminación y la aceptación de los sujetos marginales, es decir, es un arma para la lucha contra su exclusión (quizás podríamos decir, lucha de clases).

Ya mencioné que la violencia generalmente se utiliza para atacar a ciertos personajes que caracterizan a personas reales tales como los carabineros, los políticos, los de la clase alta -representantes de la masculinidad hegemónica-, así como también se ataca a los raperos falsos, los cuales son representados como mentiroso, poco confiables, influenciables por la masa y malos raperos (esto se representa en canciones como "Vete ya" de Adickta Sinfonía con Chyste Mc y "Falsos" de Gran Rah con Ley 20mil). Es fácil notar que todos los atacados son simbólicamente masculinos, por lo que la masculinidad parece ser un arma contra ellos más que un fin en sí mismo o una forma de opresión hacia la mujer.

En relación con lo anterior, es interesante ver que cuando se habla de lo femenino en el rap chileno consciente en general no se hace con agresividad; la mujer aparece en canciones de amor o comprensión (como "Amiga" de Gran rah o "Dentro de ti" de Hordatoj) y, cuando se busca atacarlas, no se le habla al tú imaginario mencionado arriba sino que se cuenta una historia (como en "Femme fatale" de Borderline) o se habla de ellas en tercera persona (como en "Las niñas blancas no tan blancas" de Chyste Mc con Cronelnegro). Además, en estos casos, es claro que no se hace una generalización hacia las mujeres, sino que se ataca a cierto sector de estas o a una en particular. Por su parte, dentro de lo hardcore ocurre que la mujer es utilizada como forma de atacar a otro hombre - "mientras me escuchas a tu mina le hacen el orto los otros seis" (Omega el Стм en Estrellas del porno, “Un Стм")-; en estos casos también se le trata como a una tercera persona y el rol parece ser más de objeto para atacar a un hombre que de objetivo en sí.

También es posible notar que dentro de las canciones, cuando se utilizan palabras que hacen referencia a la supuesta falta de hombría, como nena o maricón, estas nunca van dirigidas a mujeres u homosexuales, pues no se busca atacarlos a ellos, sino a otros hombres heterosexuales, tal y como sucede en esta cita "la hombría trajo de vuelta a patá' en la raja al que dejó a su mujer sola y embarazá" 
(Dibujo Mc en Adickta Sinfonía, "Un nuevo día”), en donde se pone en duda la masculinidad de una persona que abandona a un hijo como metáfora de su cobardía y su falta de fuerza, todo esto considerando el imaginario patriarcal.

Lo anterior demuestra que las mujeres y los homosexuales no son a los que se busca oprimir en el rap chileno, sino que su presencia corresponde más a una imagen lírica utilizada para atacar a otros hombres que cumplen con la masculinidad rapera heterosexual. Esto sucede porque la masculinidad funciona como la metáfora del poder, la cual se utiliza como una forma de empoderar a los sujetos marginales. Por lo que, se puede decir, que en las letras del rap no se busca una lucha de géneros, sino una lucha de clases o lucha contra otros raperos. Incluso muchas canciones, que intentan dar un mensaje que ayude a la sociedad, entregan su apoyo a las mujeres - "otra odisea, de la que el pueblo quiere dar respeto a la mujeres / y no por serlo, sino porque también lo eres" (arb en Adickta Sinfonía, "Un nuevo día") - y a los homosexuales -“punkys, metaleros, / anarcos y raperos / universitarios, delincuentes y extranjeros/ homosexuales y minorías en general/ podemos transformar la mierda en un bello lugar" (El tipo en Borderline, "Fuck you")-, lo que demuestra que el objetivo no es la opresión patriarcal sino que es una lucha desesperada por el poder negado, lo que no exenta al rap de ser un movimiento patriarcal.

\section{3. ¿Y qué hacer sin masculinidad? Rap femenino}

El rap chileno es una cultura muy amplia, por lo que es imposible estudiarlo cabalmente en un breve trabajo como este, aunque sea con foco en una de sus características. Por esto es que, hasta ahora, me he referido a él como si fuera un conjunto donde ciertos mecanismos masculinos ejercen una gran influencia, cayendo en una generalización que, espero se entienda, es solo una forma de acercarse, pero que no encasilla a toda la cultura. Prueba de esto es que hay muchas canciones y raperos que no proyectan esta imagen de la masculinidad mencionada, siendo el mayor ejemplo el rap femenino, por lo que veo necesario hacer una breve descripción de qué ocurre en los casos donde la masculinidad no representa una influencia tan grande como la que he expuesto.

Dentro de los raperos más famosos e influyentes podemos encontrar en su mayoría hombres, pero también encontramos mujeres, las cuales son respetadas por toda la cultura; el caso más emblemático es Ana Tijoux, rapera que perteneció a Makiza, un grupo de la segunda generación de raperos, también llamada época de oro del hip-hop chileno, que continuó haciendo música como solista luego de que el grupo se separara, siendo entonces parte también de la tercera generación o novísima escuela. Esta rapera, me aventuro a decir, es la más famosa y respetada en Chile, siendo considerada, tanto dentro como fuera de la cultura, como la persona 


\section{Adiós a las armas}

Despatriarcar América desde la cultura

(sin género) que mejor carrera ha hecho, y sin duda, de las mejores voces raperas (de nuevo, sin género) que ha tenido nuestro país. Vivo ejemplo de esto son sus presentaciones en importantes festivales como el Planeta rock 2010 o el Lollapaloza 2014 y sus nominaciones a importantes premios como el Grammy.

Además de Ana Tijoux también encontramos otras voces como Dania Neko y Michu Mc, ambas activas y respetadas en la cultura. Estas tres raperas, no siendo las únicas dentro del rap chileno, son perfectos ejemplos de cómo el rap femenino se articula sin masculinidades. Dije que las masculinidades en el rap sirven principalmente para dar poder a los raperos, el cual se usa contra la hegemonía o contra los raperos falsos. En el rap femenino, en general, no se suele atacar a los raperos falsos, sino que se enfoca en la hegemonía, adquiriendo su fuerza de la teoría feminista (como en "Antipatriarca" de Ana Tijoux) o de la teoría crítica social, muchas veces marxista (Como en "La bala" de Ana Tijoux, "Oveja negra" de Dania Neko y "Revelación revolucionadora” de Michu Mc).

La utilización de la teoría crítica social va acompañada del mismo concepto que utilizan los raperos masculinos al mostrar la dureza de la calle, pues también las raperas son hijas y sobrevivientes de esta. Es necesario recalcar que la teoría crítica social no es exclusiva del rap femenino, pues muchos raperos hombres también la usan como fuente de poder, a veces incluso complementándola con su masculinidad agresiva (como Portavoz y Adickta Sinfonía). Es importante señalar que, como se puede ver por sus características, el rap femenino está relacionado con el rap consciente y no con el hardcore, ya que el segundo no cuestiona la sociedad ni el patriarcado en ningún caso.

En relación con la estética agresiva y violenta, podemos notar que Dania Neko y Ana Tijoux no suelen utilizarla en sus canciones (lo que no quita que a veces también sean agresivas), sino que se valen de un tono más "femenino" con menos insultos y ritmos más suaves y cercanos al canto, dejando de lado toda posible masculinidad y entregando otro tipo de fuerza a sus letras. Por su parte Michu Mc sí se vale de la agresividad y la violencia, acercándose mucho al rap masculino, pero no dejando de lado su género, lo que rompe completamente los supuestos sobre ella dentro de la sociedad por ser mujer y hace que esta rapera pueda utilizar una estrategia "masculina" sin ser hombre ni aparentarlo.

Todo esto hace pensar que, a pesar de la masculinidad evidente del rap, este no es exclusivamente de los hombres ni tampoco es únicamente masculino, sino que las masculinidades son solo una estrategia para adquirir poder, es decir, una forma de empoderarse, que no desliga esencialmente a nadie, sino que, dependiendo de las circunstancias, se apodera de ciertos lenguajes que puedan ser propicios para el empoderamiento. 


\section{Conclusiones}

En nuestro país hay dos Chiles, como dice Portavoz en el otro Chile; el primero es un Chile repleto de oportunidades y luz que pertenece al poder hegemónico, pero el segundo es un Chile oculto, lleno de marginación y de pobreza; este segundo Chile vive buscando una forma de escapar del asedio constante que el primero le impone para mantener su poder, y una de las formas que ha encontrado el segundo Chile para escapar es el hip-hop, exponiendo su voz a través del rap. Sin embargo, el hip-hop es solo un medio que se le ofrece al segundo Chile, ya que no entrega herramientas para escapar y luchar contra el primer Chile, sino que se muestra como una forma de expresión. En este contexto entran las masculinidades en el rap, pues, gracias a estas, el rapero puede adquirir una de las armas usadas por la hegemonía (patriarcal) para luchar contra esta.

Cuando expongo las razones por las que el rap chileno utiliza las masculinidades como armas de poder no busco defenderlo; el rap chileno es machista y, utilizando su ultra masculinidad para adquirir poder, le da poder al patriarcado y logra que se cree más discriminación, contradiciendo su lucha contra la hegemonía. Sin embargo, tampoco creo que sea oportuno juzgarlo tajantemente y encasillarlo únicamente como un movimiento machista y patriarcal; el rap chileno es amplio y llamativo y las masculinidades son solo uno de los ejes (aunque sea un eje importante) que ayuda al rap a formarse. Incluso, como vimos, podemos encontrar rap que no es ultra masculino, sino que se basa es las llamadas teorías críticas y entrega otras herramientas de poder. En relación con lo anterior creo necesario recalcar la necesidad de estudiar más al rap y al hip-hop, no solo en el ámbito de las masculinidades sino también en la concepción de su labor social y sus posibilidades para entregar vOz y poder a los sujetos marginales.

A partir de lo anterior, este estudio podría servir como puerta de entrada para que las temáticas de género puedan ser estudiadas dentro del hip-hop en otros contextos (otras escuelas en distintos espacios o tiempos) e incluso, se podría estudiar según estos preceptos a otros movimientos cercanos al rap como el trap o el reggaetón. Por otro lado, es posible continuar este trabajo reflexionando sobre otras formas de poder y empoderamiento presentes en el hip-hop y las implicancias que estas podrían tener en cuanto a la configuración de las identidades dentro de nuestra cultura. Por último, podría tomarse la idea de la masculinidad como un arma para luchar contra cierto tipo de marginación para conocer otros movimientos igualmente masculinos como el punk o el metal.

Para concluir me gustaría recalcar una idea; el rap es una fuente de creación e investigación amplia y en constante movimiento, además de un reflejo fiel de ciertas visiones y voces de la sociedad actual que necesitan ser escuchadas, en este sentido, puede el rap ser un gran constructor del cambio si se le mira con más ojo crítico y 


\section{Adiós a las armas}

Despatriarcar América desde la cultura

se le da más importancia (esto dirigido tanto a los estudiosos de la sociedad como a los propios raperos), por lo que, si queremos una sociedad más igualitaria se debe prestar más atención a las voces y ser más cuidadoso en lo que exponen estas, y esto no solo en lo referente al hip-hop, sino que puede incluir a todas las culturas urbanas de nuestra sociedad. 


\section{Obras citadas}

Adickta sinfonía. "Identidad propia”, Larga vida al rap, Texastudio, 2012. . "Bicho raro", Larga vida al rap, Texastudio, 2012. "Un nuevo día", Larga vida al rap, Texastudio, 2012. . "Vete ya", Nacidos para esto, Texastudio, 2009.

Aerstame. "Escupo ácido", Aéreo 27, Aerstame, 2009.

Asfura, Eduardo. Cultura popular y contrahegemonía en las líricas del rap chileno independiente. Universidad de Chile, 2011.

Bascur. "Resentidos sin gloria", Piano, Piano, Bascur, 2014. ."El que la hace la paga", Piano, Piano, Bascur, 2014.

Borderline. "Femme fatale", Cortocircuito, Borderline, 2013. . "Fuck you", Morir joven, Borderline, 2011.

Chyste Mc. "Las niñas blancas no tan blancas", La paranoia del Psycho Joke Fu, Indajausman, 2010.

Codocedo, Paula. Hip Hop en Santiago de Chile. Estilo subcultural, arte y vida. Un acercamiento desde el estudio de casos de jóvenes hiphoperos. Universidad Academia de Humanismo Cristiano, 2006.

Connell, R.W. "La organización social de la masculinidad". Masculinidad/es. Poder y crisis, editado por Teresa Valdés y José Olavarría, Isis Internacional, 1997, pp. 31-48.

Da Killtros. "Uno punto nueve barrio”, Maapakn, Indajausman, 2010.

De Kiruza. "Algo está pasando”, De kiruza, Feria Music S.A., 1988.

Espitia, Juana Alexandra. "El rap es mi nación”: De representaciones y marginalidad, el barrio las cruces escenario de confluencia de conflictos. Tesis de licenciatura, Pontificia Universidad Javeriana, 2008.

Estrellas del porno. "Estilo sudaca represento", Todo un clásico, El sur es Hardcore, 2011. . "Soy un borracho", Todo un clásico, El sur es Hardcore, 2011. . "Un стм", Todo un clásico, El sur es Hardcore, 2011.

Gran Rah. "Palabras mayores", Titan, Gran Rah, 2014. . "Falsos", The end, Gran Rah, 2015. . "Vida Hardcore", Lenguaje vivo, Gran Rah, 2012. . "Perro", The real shit, Gran Rah, 2008. . "Amiga", The real shit, Gran Rah, 2008.

Hordatoj. "Dentro de ti”, El tintero, Potoco Discos, 2012. . "Un segundo", Entre lo habitual y lo desconocido, Potoco Discos, 2007.

Jonas Sanche. Estar asi en Verdades: la voz de la avenida, Jonas Sanche, 2012.

La Celda de Bob. "Mamá, Papá, soy rapero". La Celda de Bob, 18 julio 2015, http://www. laceldadebob.cl/2015/07/mama-papa-soy-rapero.html

La celda de Bob. "SIGMUND KROYS | Entrevista + Descarga | Estreno videoclip 


\section{Adiós a las armas}

Despatriarcar América desde la cultura

SUBEN". La Celda de Bob, 18 octubre 2012, http://www.laceldadebob.cl/2012/10/ sigmund-kroys-entrevista-descarga.html

Michu Mc. "Revelación revolucionadora", De mi mente a tu parlante, Michu Mc, 2011.

Movimiento Original. Soldados del ghetto, Movimiento Original, 2008.

Neko, Dania. “Casquillos”, Depura, Dania Neko, 2015. . “Oveja negra”, Depura, Dania Neko, 2015.

Omega el стм. Bienvenido al inframundo, Omega, 2012.

Poch Plá, Pedro. Del mensaje a la acción. Construyendo el movimiento hip hop en Chile. 1984-2004 y más allá. Quinto elemento, 2011.

Portavoz. Escribo rap con R de revolución, Portavoz, 2012.

Quitzow, Rainer. "Lejos de NYC: El hip hop en Chile". Bifurcaciones, no. 2, 2005, www. bifurcaciones.cl/002/Quitzow.htm

Radio Bío-bío. "Snoop Dogg afirma que la homosexualidad nunca será aceptable en el Rap" Radio Bio-bio, 7 abril 2013, http://www.biobiochile.cl/noticias/2013/04/07/snoopdogg-afirma-que-la-homosexualidad-nunca-sera-aceptable-en-el-rap.shtmlhttp:// www.biobiochile.cl/noticias/2013/04/07/snoop-dogg-afirma-que-lahomosexualidadnunca-sera-aceptable-en-el-rap.shtml

Solorzano Navarro, Héctor y Moraga González, Mario. "Cultura urbana hip-hop. Movimiento contracultural emergente en los jóvenes de Iquique”. Última Década, no 23, 2005, pp. 77-111.

Tijoux, Ana. "Antipatriarca", Vengo, Nacional Records, 2014. . "La bala", La bala, Nacional Records, 2011. 